\title{
The Effect of Iris Image Compression on Recognition Performance
}

\author{
I. J. Shaikh \\ Student M.E (E\&TC) \\ SEVERI College of Engineering Pandharpur, \\ Maharashtra, India
}

\author{
S. M. Mukane, Ph.D. \\ Professor \\ SEVERI College of Engineering Pandharpur, \\ Maharashtra, India
}

\begin{abstract}
In today's modern society automated personal identification based on biometrics has received attention not only from research community but also from industries for security applications. Iris recognition is emerging as one of the most active topics in biometrics technology because of its high reliability for identification of persons \& is a proved to be most accurate means to identify persons. Iris is consider as the most reliable biometric feature in terms of its uniqueness and robustness. For Iris Recognition iris image (eye image )is captured from different person's and these iris images should be stored in the data base \& recalled whenever required. So there is requirement of large databases of iris images. If available storage space is not enough for these images, compression will be an option. Compression allows a reduction in the space needed to store these iris images. The objective of this paper is to present the effects of iris image compression on the recognition performance. Normally, iris images are 600 times bigger than the Iris Code templates which required larger space for storage .But it is expected that iris data should be stored, transmitted, and embedded in media in the form of images rather than as templates. To obtain this goal with its implications for bandwidth and storage, this paper present the scheme that combine region-of-interest isolation with JPEG ,JPEG 2000 \& lifting based wavelet compression at different levels using publicly available database of iris images. It is concluded that lifting wavelet compression gives the better result as compared to JPEG \& JPEG 2000 with minimum impact on recognition performance.
\end{abstract}

\section{General Terms}

Iris Recognition, Security purpose , Algorithms, Recognition performance, compression

\section{Keywords}

JPEG, JPEG 2000 , Lifting wavelet ,Iris template ,Hamming Distance(HD) ,Region of Interest (ROI), Cropping .

\section{INTRODUCTION}

With the increasing demand of biometric systems the question arises naturally that how to store and handle the obtained sensor data. So, the compression of these data may become important under certain circumstances due to the large amounts of data involved. The compression technology may be used in two stages of the processing chain in classical biometric recognition[1].

\subsection{Storing the Reference Data}

In most template databases (where the reference data of the enrolled individuals are stored) only the extracted features which are required for the matching step are stored as compare with originally acquired sensor data. But governments, regulatory bodies, and international standards organizations specify that biometric data should be recorded and stored in a raw form, rather than in (or in addition to) post-processed templates. Storing of the original sensor data solves this problem. But these data should be stored in compressed form to save the storage space and also in encrypted (to protect privacy) form[1].

\subsection{Data Transmission after Sensor Data Acquisition}

In distributed biometric systems, the data acquisition stage is normally separated from the feature extraction and matching stage. In such situation the sensor data have to be transferred via a network link to the respective locations, normally over wireless channels with low bandwidth and high latency. Therefore, a minimization of the amount of data to be transferred is desired, which is achieved by compressing the data before transmission. A alternative solution is to extract the features before transmission and to transfer feature data only, but feature extraction is more complicated as compared to compression which generates additional workload. So, the compression of the raw sensor data can be advantageous in many applications, we have to identify techniques suited to perform this task in an optimal manner. In order to maximize the benefit in terms of data reduction compression techniques have to be applied[1]

This paper, investigate that the raw image data can be compressed without significantly affecting the biometric templates computed from the data and it is found a rough estimation of template and image data sizes with minimum impact on iris recognition performance. Compression in biometrics is also important because governments, regulatory bodies, and international standards organizations expected that biometric data must be recorded and stored in a raw form, rather than in (or in addition to) post-processed templates. Enrolling or storing raw image data also important which made these databases more "future-proof" since they can benefits the future improvements in recognition algorithms, simply by enrolling a new the raw data. Also, the directive for, standards bodies like ISO [2] and industry consortia such as RTIC [3] that embed biometric data into smart cards is to avoid incorporating patented techniques into data formats and Standards. But storing raw images instead of templates can increases the size of data hundred times, with problems such as increased data transmission times and inability to fix the raw data in the allocated Space in smart cards, which in the case of the RTIC specification [3] for iris images is a about 4,000 bytes per eye. So the questions of iris image compression and its effects on recognition performance become critical.

In this paper JPEG, JPEG 2000 \& Lifting wavelet compression schemes are study for iris image compression that retain rectilinear image formats but achieve severe 
compression at different levels, while still allowing very good recognition performance on the CASIA publicly available iris image database.

\section{JPEG COMPRESSION}

JPEG (Joint Photographic Experts Group) is an international compression standard for continuous-tone still image, both grayscale and color.JPEG algorithm start with dividing the image in $8 \times 8$ block \& than DCT is applied. After application of DCT quantization matrix is used \& than zigzag ordering .The Last step in JPEG compression is entropy coding.JPEG transformation is based on Discrete Cosine Transformation.[4]

\section{JPEG 2000 COMPRESSION}

It was created by the Joint Photographic Experts Group committee in 2000 with the intention of improvement over their original discrete cosine transform-based JPEG standard (created in 1992) with a newly designed, wavelet-based method. The JPEG 2000 standard offer more flexible modes of use, and achieving typically $20-30 \%$ more compression at any given image quality .Mathematically based on a Discrete Wavelet Transform (DWT) onto Daubecies wavelets rather than the Discrete Cosine Transform (DCT), JPEG2000 is not suffering as badly from the block quantization artifacts which was drawback of JPEG at low bit-rates. This block artifacts in JPEG was due to the fact that the DCT simply chops cosine waves inside box windows with obvious truncation consequences when they are sparse (not dense) and incomplete. Also, the different levels within the multiresolution DWT wavelet decomposition allow local areas in each image tile to be encoded using different subbands of coefficients as needed[5]. The net superiority of JPEG2000 over JPEG in terms of image quality especially marked at the very low bit-rates corresponding to high compression. Different mechanisms exist within JPEG2000 for heterogeneous allocation of the coding budget, including tile definition, code-block selection allowing different DWT resolution levels in different tiles, and DWT coefficient scaling.

\section{LIFTING WAVELET COMPRESSION}

The Wim Sweldens developed the lifting scheme for the construction of biorthogonal wavelets[6]. The main advantage of the lifting scheme is that all constructions are done in the spatial domain. It does not used complex mathematical calculations that are used in traditional methods. Lifting scheme is simple and efficient algorithm to calculate wavelet transforms. Lifting scheme is used to generate secondgeneration wavelets, which are not necessarily translation and dilation of one particular function [6]. Earlier it was started as a method to improve given discrete wavelet transforms to obtain specific properties, but later it become an efficient algorithm to calculate any wavelet transform as a sequence of simple lifting steps. Lifting scheme consists of mainly three steps:[6]

$>$ In first step there is splitting of phase that split data into odd and even sets.

$>$ The second step is predict step. In this step odd set is predicted from even set. Predict phase ensures polynomial cancellation in high pass.

$>$ The third step is update phase that will update even set using wavelet coefficient to calculate scaling function. Update stage ensures preservation of moments in low pass.
The Inverse wavelet process is exactly opposite of forward process. i.e. Undo update, Undo predicate, \& Merge. In this paper along with JPEG \& JPEG 2000 lifting scheme of wavelet transform for the compression is used because lifting scheme is having following advantages over conventional wavelet transform technique.[7]

- As compare to classical Discrete wavelet transform, lifting based scheme required half number of computation .So Lifting wavelet allow faster implementation of the wavelet transform.

- The lifting wavelet scheme uses a fully in-place calculation of the wavelet transform. That Means, no auxiliary memory is needed and the original signal can be replaced with its wavelet transform.

- In lifting scheme reversible integer wavelet transforms is used as compare to conventional scheme which involves floating point operations, which produces rounding errors due to floating point arithmetic.

- In lifting scheme perfect reconstruction is possible for loss less compression.

- Lifting scheme is easy to understand and implement.[7]

The basic idea of the lifting scheme is to break up the polyphase matrices for the wavelet filters into a sequence of upper and lower triangular matrices and then converting the filter implementation into banded matrix multiplication.

\section{PROPOSED WORK}

In this paper first original image template is extracted. After that this original image is compressed by using different compression factor for JPEG, JPEG 2000 \& Lifting wavelet scheme to obtain their templates. Then the original image templates with different compressed image templates are compared using Hamming Distance (HD) method. Based on this comparison the recognition performance is judged .

\subsection{Simple Cropping}

First step is to reduce image size from the standard iris image format of $320 \times 280$ pixels with 8 bits grayscale data per pixel, consuming 89600 bytes, smaller size containing the iris. The cropping is done manually to localize the iris in all images with the iris centered in it. The new gallery of grayscale eye images with this is obtained \& then JPEG, JPEG 2000 \& lifting wavelet compression is performed for various compression factors. Figure 1 shows original image where as figure 2 shows cropped iris image.

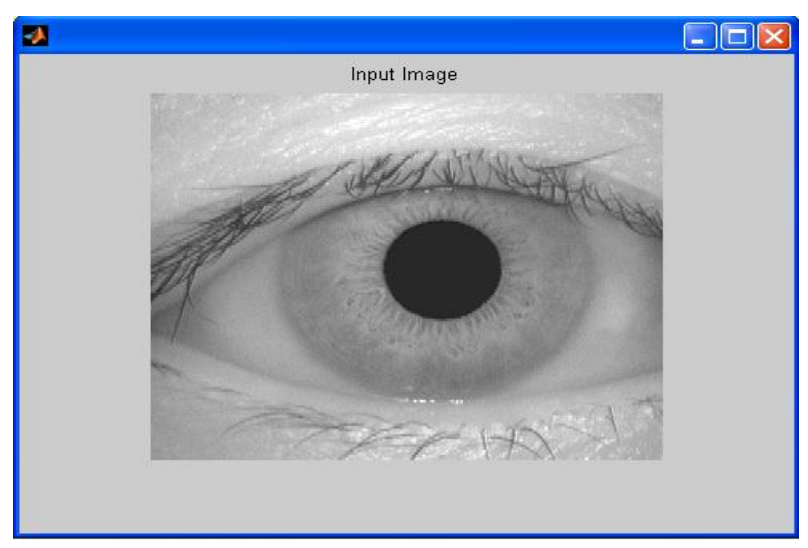

Figure 1 Original Eye Image 


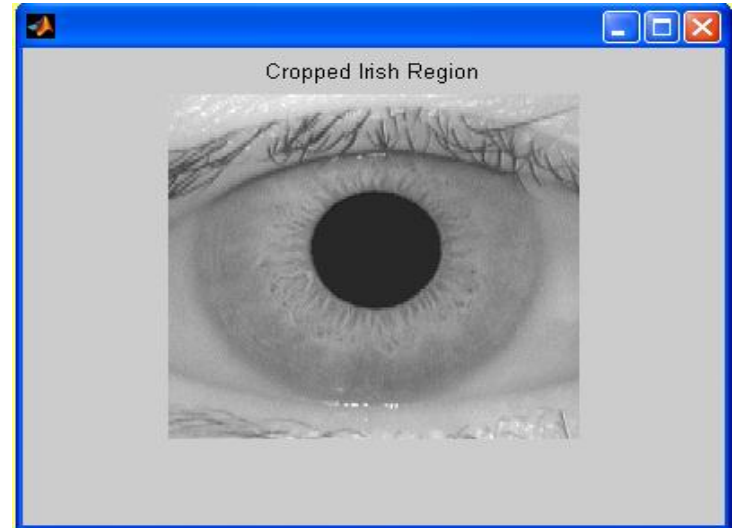

Figure 2 Cropped Eye Image

\subsection{Region-of-interest (ROI) Segmentation}

The JPEG, JPEG 2000 \& Lifting scheme compression can be made more effective if we replaced all non-iris parts of the image with a uniform gray value. This kind of substitution of pixel values within a rectilinear image array is preferable, from the viewpoint of Standards bodies, than actual extraction and mapping of pixel data from a normalized ("unwrapped") iris because it is desirable to be as shape-agnostic and as algorithm-neutral as possible[8]. All compression schemes study here fits themselves well with region-of-interest (ROI) differential assignment of the coding budget [8]. This idea was used for biometric face recognition by Hsu and Griffin , who demonstrated that recognition performance was degraded by only $2 \%$ for file sizes compressed to the range of $10,000-$ 20,000 bytes with ROI specification. Here it is investigate that how much compression of iris images can be achieved with minimal impact on iris recognition performance, using the ROI idea without "unwrapping" the iris but retaining a rectilinear pixel array format. Figure 3 shows the cropped image same as figure 2 but now with region of interest (ROI) process [8] .Non-iris regions must be encoded in a way that distinguishes sclera from eyelids or eyelashes regions, so that post-compression algorithms can still determine both type of iris boundaries.

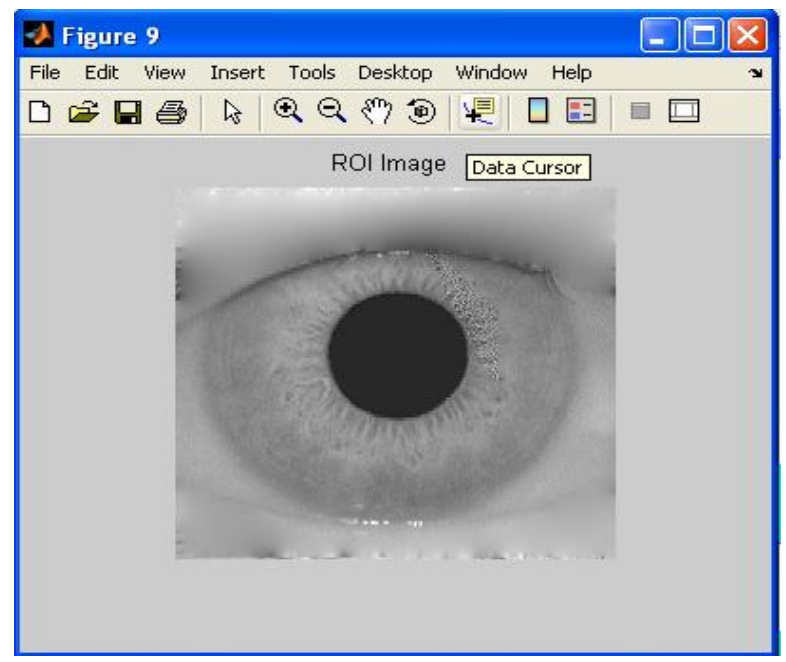

Figure 3 Cropped Eye Image with ROI

Since the substitution gray levels are uniform, their coding cost is minimum and could be further reduced by using larger data units. For any given specified CF, the result of iris ROI isolation is nearly a two-fold reduction in file size while maintaining a simple rectilinear image format and easy localization of eyelid boundaries in later stages. Substitution of non-iris regions by uniform gray levels prevents wasting the coding budget on costly irrelevant structures such as eyelashes. [8]

\section{RESULT AND DISCSSION}

The effect of iris image compression on recognition performance by using JPEG, JPEG 2000 \& Lifting wavelet scheme as a compression algorithm is investigated. Based on certain threshold value for comparing two iris templates it can be concluded that the compressed images are recognized or not. The Hamming distance gives a measure of number of bits that are same between two bit patterns. Using the Hamming distance of two bit patterns, a decision is made about recognition of patterns

Biometric recognition performance is measured by generating ROC (Receiver Operating Characteristic) curves, which plot the trade-off between two error rates viz False Accept Rate(FAR) and False Reject Rates(FRR) also called False Match and False non- Match Rates. The decision threshold for similarity scores is varied from conservative to liberal. It is common to tabulate specific points on such trade-off curves, such as the FRR when the decision threshold causes an FAR of 1 in 1,0000 or of 1 in 1,000 and the point at which the two error rates are equal, FRR $=$ FAR $=$ EER, the Equal Error Rate.[8]

\subsection{ROC curve of JPEG, JPEG 2000, and Lifting scheme}

\subsubsection{ROC of JPEG compression}

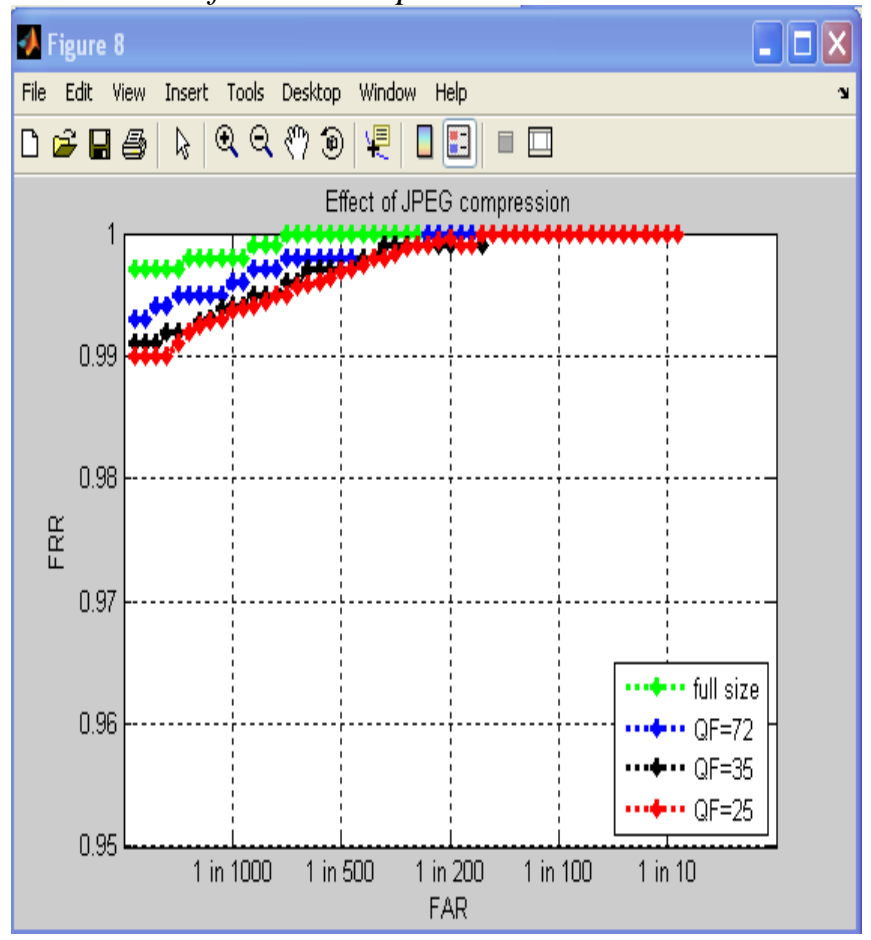

Figure 4: ROC curves for different quality factor for JPEG Compression

Figure 4 shows the ROC curve obtained for JPEG compression. Green line shows the performance of original image, blue line shows the performance of compressed image at quality factor of 72, black line shows performance of compressed image at quality factor of $35 \&$ red line shows performance of compressed image at quality factor of 25 . It has been observed from ROC curve that the recognition 
performance is degraded if we decrease quality factor for JPEG compression.

\subsubsection{ROC of JPEG 2000 compression}

Figure 5 shows the ROC curve obtained for JPEG 2000 compression. Green line shows the performance of original image, blue line shows the performance of compressed image at compression factor of 25 , black line shows performance of compressed image at compression factor of $35 \&$ red line shows performance of compressed image at compression factor of 72. It has been observed from ROC curve that recognition performance is degraded if we increase compression factor. The recognition performance is improved if we use JPEG 2000 instead of JPEG algorithm. Also JPEG 2000 algorithm provides higher compression as compare to JPEG.

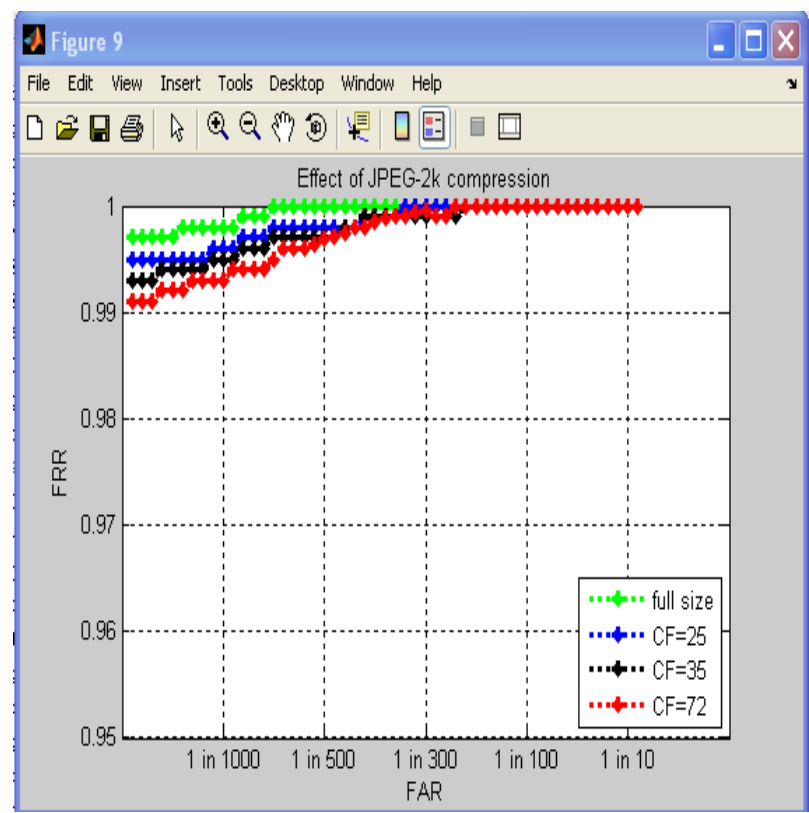

Figure 5: ROC curves for different quality factor for JPEG 2000 Compression

\subsubsection{ROC of lifting wavelet compression}

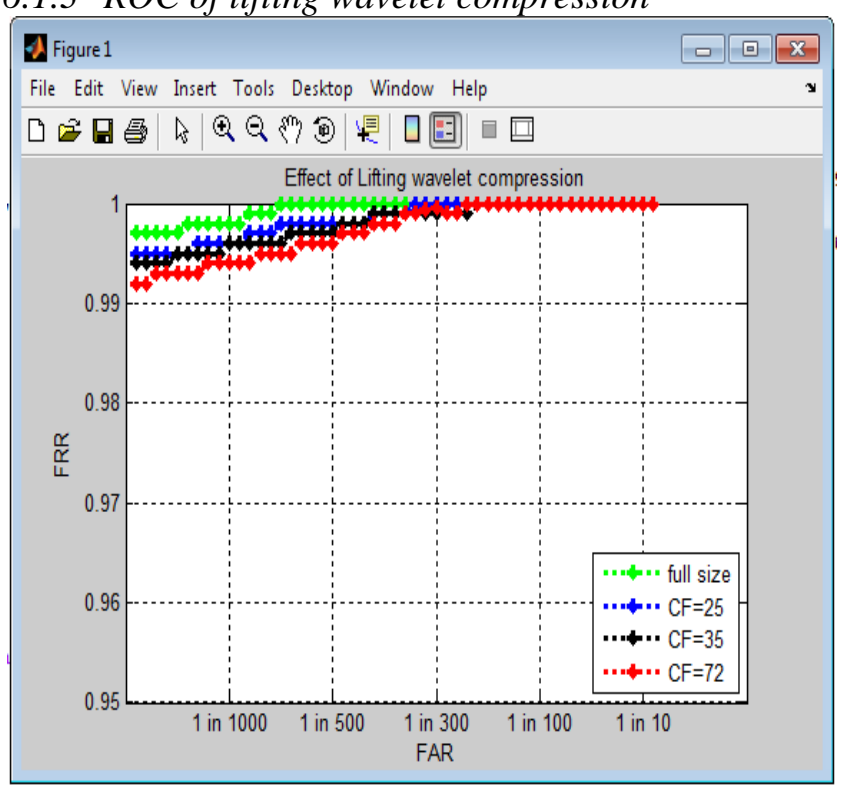

Figure 6: ROC curves for different compression factor for Lifting wavelet Compression
Figure 6 shows the ROC curve obtained for Lifting wavelet compression. Green line shows the performance of original image, blue line shows the performance of compressed image at compression factor of 25 , black line shows performance of compressed image at compression factor of $35 \&$ red line shows performance of compressed image at compression factor of 72. It has been observed from ROC curve that recognition performance is degraded if we increase compression factor .As compare to JPEG, JPEG 2000 Lifting scheme provide better recognition performance as well as higher compression rate with minimum impact on recognition performance

\subsection{ROC curve of JPEG, JPEG 2000, and Lifting wavelet with ROI}

\subsubsection{ROC curve of JPEG compression with ROI}

Figure 7 shows the ROC curve obtained for JPEG compression with ROI . As seen from ROC curve we can analyze that performance will remain somewhat same as without ROI but advantage of ROI is that it reduces the size of iris image two times as compare to without ROI . It has been also observed from ROC curve that the recognition performance is degraded if we decrease quality factor for JPEG compression.

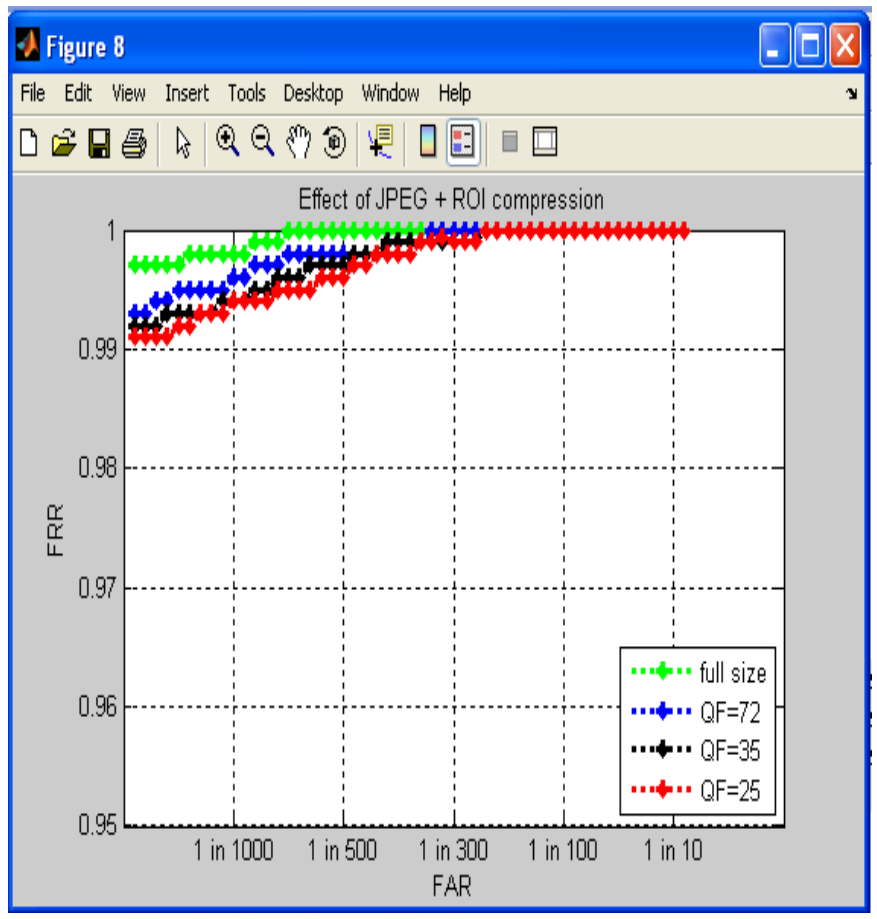

Figure 7: ROC curves for different quality factor for JPEG Compression with ROI

\subsubsection{ROC curve of JPEG 2000 compression with \\ ROI}

Figure 8 shows the ROC curve obtained for JPEG 2000 compression with region of interest (ROI).It can be analyzed from ROC curve that recognition performance is degraded if we increase compression factor. From ROC curve we can analyze that performance will remain somewhat same as without ROI but advantage is that it reduces the size if iris image two times as compare to without ROI. As compare to JPEG, JPEG 2000 algorithm provides better recognition performance $\&$ also higher compression rate. 


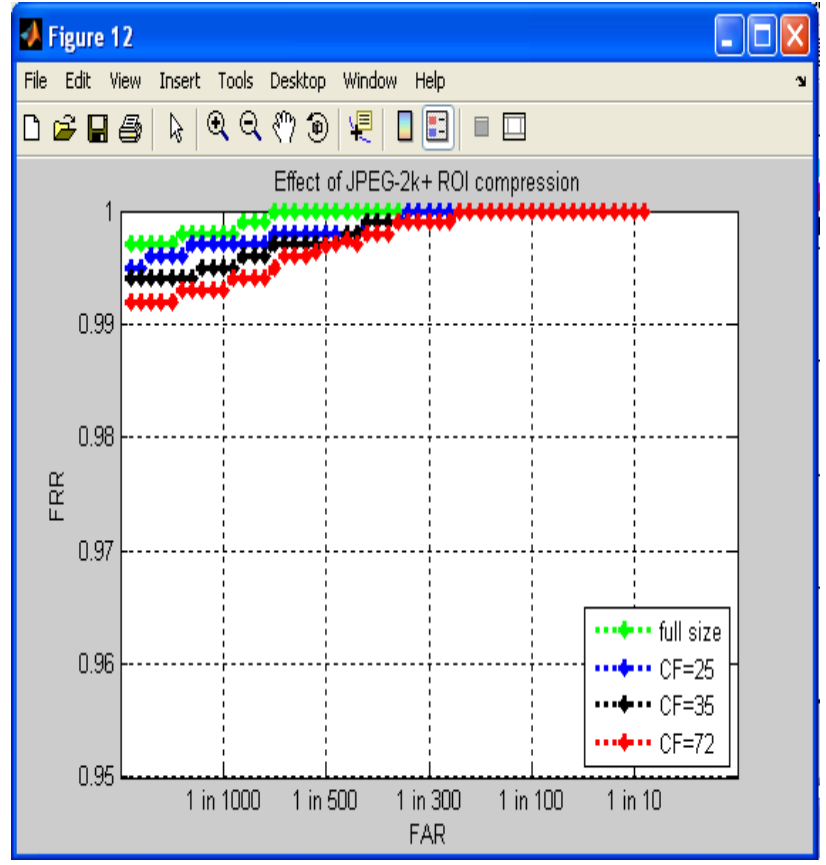

Figure 8: ROC curve for different compression factor for JPEG 2000 compression

\subsubsection{ROC curve of Lifting wavelet compression} with ROI

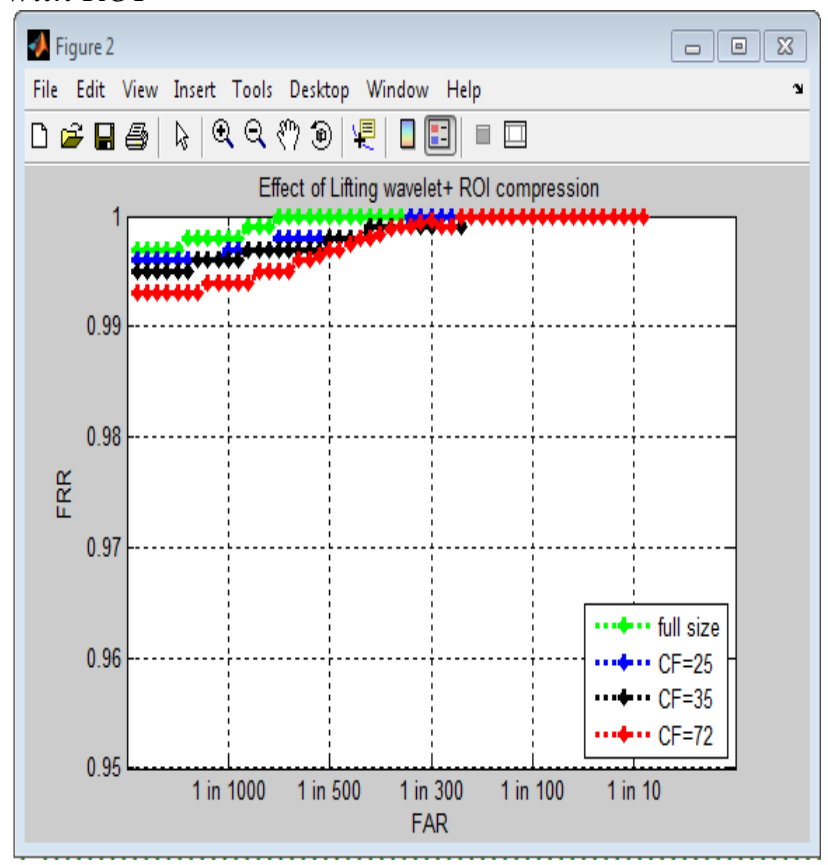

Figure 9: ROC curve for different compression factor for Lifting wavelet compression

Figure 9 shows the ROC curve obtained for lifting wavelet compressed image with region of interest (ROI). It can be analyzed from ROC curve that performance degraded if we increase compression factor. From ROC curve we can analyze that performance will remain somewhat same as without ROI but advantage is that it reduces the size if iris image two times as compare to without ROI.As compare to JPEG \& JPEG 2000 algorithm the lifting wavelet scheme provide better recognition performance $\&$ also higher compression rate.

\section{CONCLUSION}

In this paper effect of image compression on iris recognition performance by using JPEG, JPEG 2000 \& lifting wavelet as compression algorithm is studied. Hamming Distance of each compressed image with original image is compared which leaded to the conclusion that even severely compressed image using different quality factor remains perfectly serviceable.It is important to use region-of-interest(ROI) isolation of the iris within the image so that the coding budget is allocated almost to the iris. The Iris Codes generated under each method have been compared with the corresponding original uncompressed images with the help of hamming distance and impact is studied. The average Hamming Distance between such IrisCodes obtained before and after image compression indicate that only about $2 \%$ to $3 \%$ of the IrisCode bits change as an effect of image compression even though the image is compressed to very small size. Out of three compression schemes used the lifting wavelet gives the best performance in term of ROC curve. It is concluded that lifting wavelet compression scheme provide better recognition performance. As a feature scope we can apply these compression algorithm at different levels in iris processing chain and then observe what would be there impact on recognition performance.

\section{REFERENCES}

[1] RobertW. Ives,1 Daniel A. Bishop,2 Yingzi Du,3 and Craig Belcher3" Iris Recognition: The Consequences of Image Compression" Hindawi Publishing Corporation EURASIP Journal on Advances in Signal Processing Volume 2010 Article ID 680845, 9 pages

[2] International Standard ISO/IEC 19794-6: "Information technology - Biometric data interchange formats - Part 6: Iris image data." ISBN 0-580-46456-3, 2005.

[3] Registered Traveler Interoperability Consortium (RTIC). "Technical Interoperability Specification for the Registered Traveler Program (Version 1.0 - Final)," 2006. Available at: http://www.rtconsortium.org/

[4] R.L.V. Hsu and P. Griffin, "JPEG region of interest compression for face recognition," IDENTIX Document No. RDNJ-04-0102, 2005.

[5] A.P. Bradley and F.W.M. Stentiford, "JPEG2000 and region of interest coding," Digital Imaging Computing Techniques and Applications, Melbourne, 2002. Available at: http://www.ee.ucl.ac.uk/ fstentif/DICTA02.pdf

[6] Lifting wave let information available on http://shodhganga.inflibnet.ac.in/bitstream/10603/4341/7/ 07_chapter\%203.pdf

[7] M.S. Abdullah, N. Subba Rao" Image Compression using Classical and Lifting based Wavelets" International Journal of Advanced Research in Computer and Communication Engineering Vol. 2, Issue 8, August 2013

[8] J. Daugman \& cathryn Dowing, "Effect of Serve Image compression on .iris recognition performance ," IEEE Trans. on Information \& Security, March, 2008.

[9] International Standard ISO/IEC 10918: "Information technology - Digital compression and coding of continuous-tone still images," 1994. http:www.jpeg.org 
[10] International Standard ISO/IEC 15444-1: "Information technology - JPEG 2000 image coding system," 2004. Available at: http://www.itu.int/rec/T-REC-T/en

[11] C. Christopoulos, A. Skodras, and T. Ebrahimi, "The JPEG2000 still image coding system: an overview," IEEE Trans. Cons. Electr., vol. 46, no. 4, pp. 1103-1127, 2000. Available : http://jj2000.epfl.ch/jj publications/papers/006.pdf

[12] R.L.V. Hsu and P. Griffin, "JPEG region of interest compression for face recognition," IDENTIX Document No. RDNJ-04-0102, 2005

[13] L. Ma, T. Tan, Y. Wang, and D. Zhang, "Efficient iris recognition by characterizing key local variations,"
IEEE Trans. Image Processing, Vol. 13, no. 6, pp. 739 750, June 2004.

[14] A. P. Bradley and F. W. M. Stentiford, "JPEG2000 and region of interest coding," in Digital Imaging Computing Techniques and Applications, Melbourne, Australia, 2002 [Online].Available: http://www.ee.ucl.ac.uk/ fstentif/DICTA02.pdf.

[15] Robert W.Ives, Randy p.Broussard ,Lauuren R.Kennell , David I.Soldan " Effect of image compression on Iris Recognition system performance "Journal of Electronic Imaging17(1).011015(Jan-Mar 2008)

[16] Robert W.Ives , Daniel A.D.Bishope, Yingzi Du,\& Craig Belcher" Effect of image compression on Iris Recognition performance and image quality "IEEE 2009 